\title{
Resource Allocation Method of Device-to-Device Communication
}

\author{
Wenji Feng, Yafeng Wang, Lei Yang \\ Wireless Theory \& Technology lab (WT\&T), Beijing University of Posts and Telecommunications, Beijing, China \\ Email: fwj10171017@sina.com,wangyf@bupt.edu.cn, yanglei5658@gmail.com
}

Received July, 2013

\begin{abstract}
In this paper, we study D2D (Device-to-Device) communication underlying LTE-Advanced uplink system. Since D2D communication reuses uplink resources with cellular communication in this scenario, it's hard to avoid the inference between D2D users and cellular users. If there is no restriction for D2D communication on using the whole uplink frequency band, it will have a strong negative impact on cellular communication. In order to overcome this shortage, we propose a resource allocation method that D2D users and cellular users use orthogonal frequency resources. This method will effectively reduce the inference between both kinds of communication. However, an obvious disadvantage of this method is no effective use of uplink resources. Based on this, we propose an optimized resource allocation method that a specific cellular user will be chosen to reuse the RBs (Resource Block) of D2D users. These ideas will be taken into system-level simulation, and from the results of simulation we can see that the optimized method has the ability to improve overall system performance and limit inference for cell-edge users.
\end{abstract}

Keywords: Device-to-device Communication; LTE-Advanced System; Resource Allocation Method; up Link; Cell-edge Users

\section{Introduction}

3GPP Long Term Evolution (LTE) technology has been proved to have outstanding performance, especially in the measures of spectral efficiency and the average throughput, cell-edge and peak values in a cellular, frequency reuse one network [1,2]. Hence, major efforts have been spent on the development of LTE. Currently the further evolution of such systems has been started under the scope of LTE-Advanced [3-5].

The device-to-device communication (D2D) technology, also known as proximity-based services (ProSe), is introduced into LTE-Advanced system [6-7]. However, D2D communication is sharing authorized frequency band with cellular communication by the way of orthogonal method or multiplexing method. The introduction of D2D communication is to improve the throughput of overall cellular system. It contributes to higher frequency resource utilization as well. When D2D users reuse cellular frequency resources, it is hard to avoid the interference of other cellular users in cell. It may affect the D2D user's communication quality to some extent. If there are no restrictions for D2D communication on using the whole frequency band, it will have a strong negative impact on cellular communication.

As mentioned above, appropriative resource allocation methods of D2D communication may improve this situa- tion. Because of frequency reusing with cellular communication, D2D communication should choose a better resource allocation method to avoid inference against each other. Hence, we hope to find out simulation scenarios of different resource allocation methods so that we can choose an appropriative allocation method for D2D communication.

This paper is organized as follows: Section 2 describes the simulation platform. Section 3 shows different kinds of D2D communication resource allocation methods, and the performance will be analyzed in this section. According to section 3 , we propose some optimization method of D2D resource allocation method in section 4 . Finally concluding remarks are made in Section 5.

\section{Simulation Platform}

Assume that the D2D communication underlying LTEAdvanced network only reuse uplink frequency resource. The simulation platform consists of 7 eNB (21 sectors). We distribute one pair of D2D UEs into one cell amount to 21 pairs of D2D UEs. In our simulation platform, we consider that there is one transmitter and one receiver in a pair of D2D UEs, which are working only during uplink slot. The distribution of eNB (evolved node B) and D2D UEs is shown in Figure 1. 


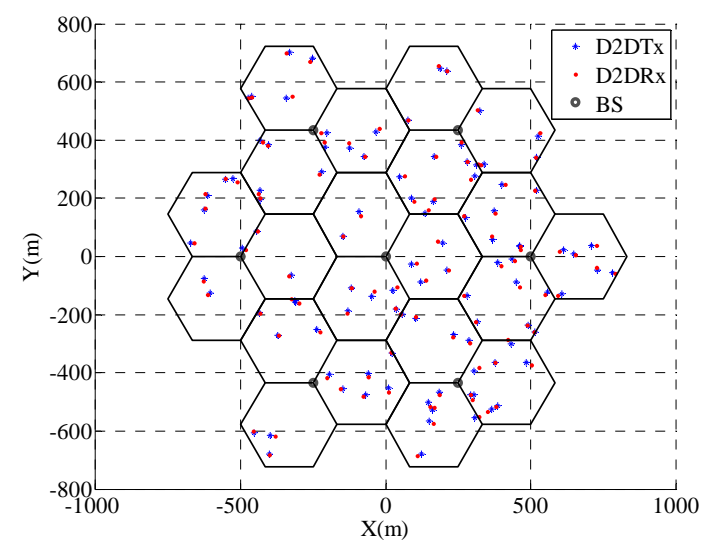

Figure 1. Distribution of eNB and D2D.

The radius of each cell is $500 \mathrm{~m}$. The distance between transmitter and receiver is $10 \mathrm{~m}$ to $20 \mathrm{~m}$. D2D communication reuses uplink resources of LTE-Advanced, which contains $46 \mathrm{RB}$. The transmission power [8,9] of D2D transmitter is $20 \mathrm{dBm}$. The system bandwidth is $10 \mathrm{MHz}$ and the carrier frequency is $2 \mathrm{GHz}$. We distribute 210 cellular UEs into overall system so that there are 8 to 12 cellular UEs in each cell. The per-manent MCS of D2D communication is 28. The path loss model and corresponding shadow fading model of D2D communication are referred as model of Urban Macro (UMa) in [10], where it can be further categorized as Line-of-sight (LOS) and Non-line-of-sight (NLOS).

LOS:

$$
P L=16.9 \log _{10}(d[m])+46.8+20 \log _{10}\left(f_{c}[G H z] / 5.0\right)
$$

NLOS:

$$
P L=40 \log _{10}(d[k m])+30 \log _{10}\left(f_{c}[M H z]\right)+49
$$

The probability of LOS is as follow:

$$
P_{L O S}=\left\{\begin{array}{cc}
1, & d \leq 4 \\
\exp (-(d-4) / 3), & 4<d<60 \\
0, & d \geq 60
\end{array}\right.
$$

Where $P L$ denotes the path loss, $d$ is the distance between D2D users, $f_{c}$ is carrier frequency, and $P_{\text {LOS }}$ is the probability of LOS.

The parameters of simulation are listed in Table 1.

\section{Resource Allocation Method}

\subsection{Resource Allocation Method of D2D}

In order to analysis the performance of different methods of D2D resource allocation, first of all we assume Case Basic, which is a LTE-Advanced uplink network without D2D communication. In this simulation scenario, we set D2D transmission power to fixed $20 \mathrm{dBm}$, and the MCS will be the highest one. On this basis, we propose another
3 cases of D2D resource allocation method, which are described below:

a) Case Basic:

This case is LTE-Advanced uplink system without D2D communication.

b) Case All_RB_Reuse:

As Figure 2 shows, D2D communication reuses all uplink frequency resource with cellular system.

c) Case 10_RB_Reuse:

As Figure 3 shows, D2D communication reuses 10 RB of cellular uplink frequency resource. Cellular UEs still use all uplink frequency resource.

d) Case 10_RB_Sep:

As Figure 4 shows, D2D communication uses 10 RB of uplink frequency resources. Cellular UEs only use another $36 \mathrm{RB}$ of uplink frequency resource. It means that the RB of D2D and cellular is orthogonal.

Table 1. List of simulation parameters.

\begin{tabular}{ll}
\hline Parameters & Value \\
\hline Number of eNB & 7 eNB (21 sectors) \\
Radius of cell & $500 \mathrm{~m}$ \\
$\begin{array}{l}\text { Distance of D2D } \\
\text { communication }\end{array}$ & $10 \mathrm{~m}$ to $20 \mathrm{~m}$ \\
System resources & Uplink \\
Transmission power of D2D & $20 \mathrm{dBm}$ \\
System bandwidth & $10 \mathrm{MHz}$ \\
Carrier frequency & $2 \mathrm{GHz}$ \\
Number of cellular UE & 210 \\
Number of D2D pairs & 21 \\
Distribution & 8 to 12 cellular UEs in each cell; \\
MCS of D2D & one pair of D2D-UE into one cell \\
Thermal noise density & 28 \\
Scheduling algorithm & -174 dBm/Hz \\
\hline
\end{tabular}

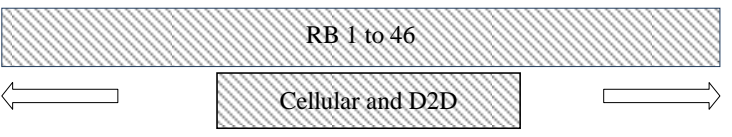

Figure 2. Caes All_RB_Reuse.

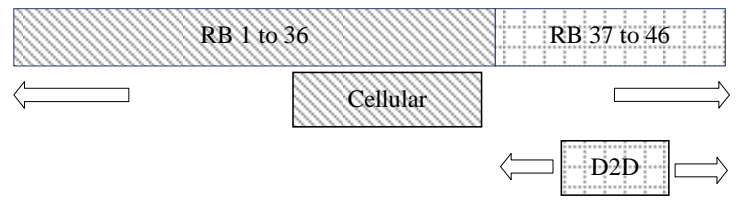

Figure 3. Case 10_RB_Reuse.

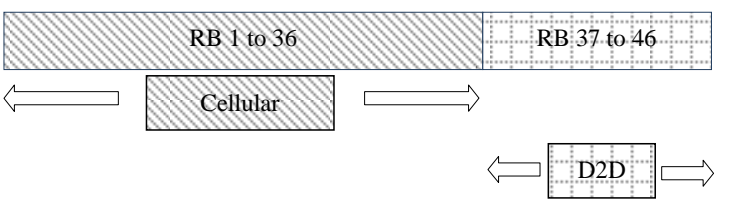

Figure 4. Case 10_RB_Sep. 


\subsection{Performance Analysis}

We compare the simulation results of previous 4 cases including average throughput of eNB, throughput of cell-edge users, throughput of D2D communication, throughput of overall system and BLER (Block Error Rate) of D2D communication. Figure 5 illustrates the simulation results of 4 cases.

From Figure 5, we recognize that if D2D communication reuses all resource with cellular communication, the throughput of overall system gets a very large increase. However, on the other hand, D2D communication brings a lot of interference against cellular communication so that cell-edge users almost unable to transmit data correctly. At the same time, because of interference from D2D communication, the throughput of cell centre users drop sharply. Therefore, if there are no restrictions for D2D communication on using the whole frequency band, it will have a strong negative impact on cellular commu- nication [11,12], which is not appropriate in the practical communication system.

In the view of this, D2D communication only use part of uplink frequency resource. In Case 10_RB_Reuse, D2D communication only reuses $10 \mathrm{RB}$ with cellular system, and the all $46 \mathrm{RB}$ is still used by cellular. In this simulation case, comparing with Case All_RB_Reuse, cellular system throughput gets a large increase, but the throughput of cell-edge UEs is still very low so that celledge users cannot communicate normally.

At the same time, we consider Case 10_RB_Sep, which D2D communication uses $10 \mathrm{RB}$ of cellular uplink frequency resource, and cellular UEs only use the other $36 \mathrm{RB}$ of uplink frequency resource. From the results of simulation, the performance of Case 10_RB_Sep is the most equilibrium. What's more, comparing with Case Basic the throughput gets a lot of gain, and the BLER of D2D is the lowest.
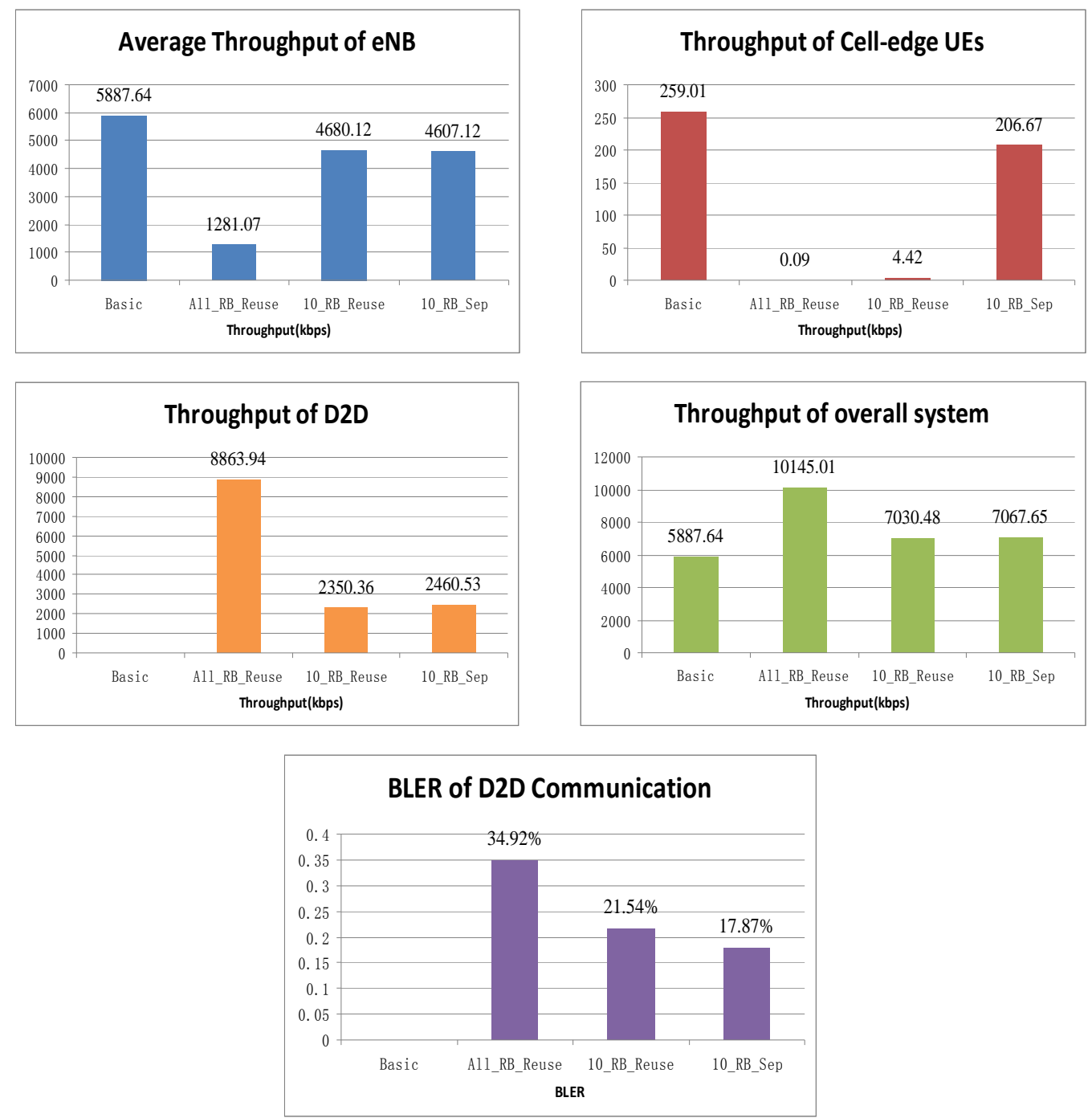

Figure 5. Results of four simulation cases. 
Figure 6 shows the cellular users SINR of 4 cases.

From Figure 6, we can still have the conclusions as above. In Case All_RB_Reuse, the SINR of cellular UEs is sharply decreased. It causes the exacerbation of overall system. In Case 10_RB_Reuse, the effects of SINR are more embodied at cell-edge users. The throughput of cell-edge UEs is still very low so that cell-edge users cannot communicate normally in this case. In Case 10_RB_Sep, the performance of SINR is almost the same as Case Basic.

\section{Optimization Scheme}

\subsection{Optimization Simulation Scenarios}

We describe Case 10_RB_Sep in section 3, which has the best performance of all 4 cases. However, in Case 10_RB_Sep, cellular communication gives up $10 \mathrm{RB}$ to assure the excellent performance of both cell-edge users and D2D communication. The $10 \mathrm{RB}$, which are given up by cellular communication and only used by D2D communication, still negatively affect the performance of overall system. Hence, we hope to find a possible way to overcome the shortage. On the basis of Case 10_RB_Sep, we consider choosing one cellular UE to reuse $10 \mathrm{RB}$ with D2D UEs. The simulation scenarios are described as follow:

a) Case 10_RB_Sep:

This case is described in section 3.

b) Case Random_UE:

We choose a random cellular UE from current cell to
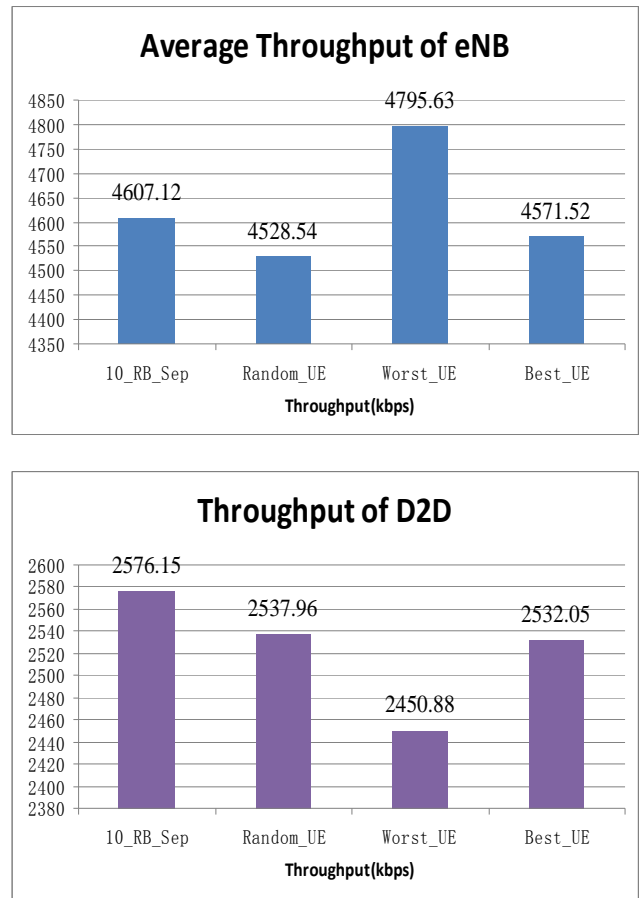

reuse $10 \mathrm{RB}$ with D2D communication. The rest of cellular users still use the other $36 \mathrm{RB}$.

c) Case Worst_UE:

We choose a cellular UE from current cell, which has the slowest transmission rate currently, to reuse $10 \mathrm{RB}$ with D2D communication. The rest of cellular users still use the other $36 \mathrm{RB}$.

d) Case Best_UE:

We choose a cellular UE from current cell, which has the highest transmission rate currently, to reuse $10 \mathrm{RB}$ with D2D communication. The rest of cellular users still use the other $36 \mathrm{RB}$.

\subsection{Performance Analysis}

We propose 3 cases to compare with Case 10_RB_Sep, the results of performance are shown in Figure 7 .

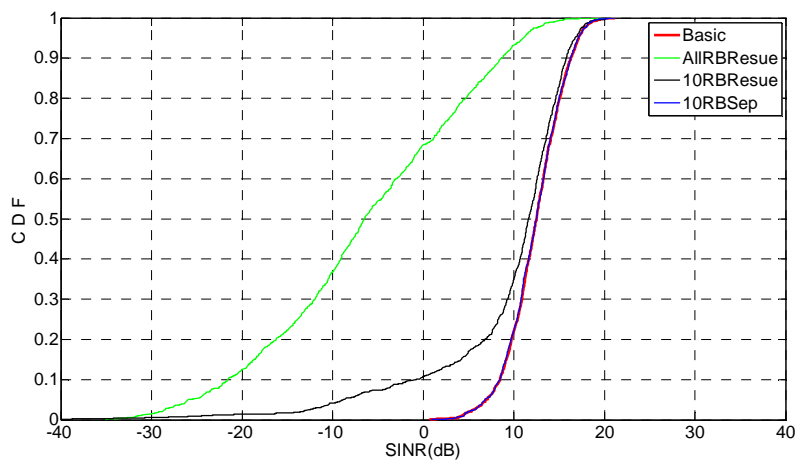

Figure 6. Cellular UEs SINR.
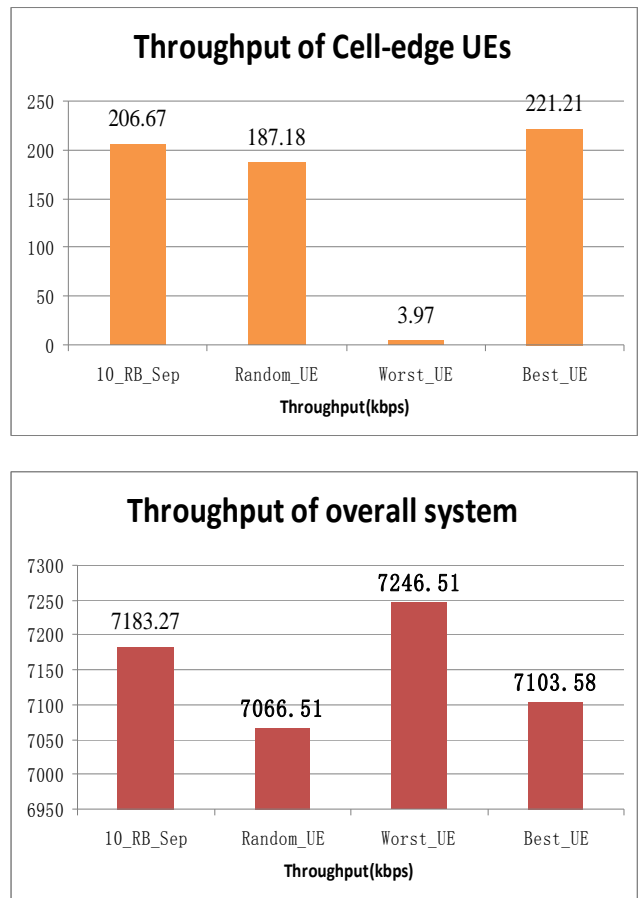

Figure 7. Results of optimized simulation cases. 
From Figure 7, we can draw the following conclusions:

In Case Random_UE, the throughput of eNB, D2D and overall system is all lower than Case 10_RB_Sep, and the throughput of cell-edge users also lower than Case 10_RB_Sep. So Case Random_UE is not an appropriate case to further optimize the performance.

In Case Worst_UE, the throughput of eNB and overall system increases a lot, but the throughput of D2D communication fall sharply. And worst yet, the throughput of cell-edge UEs is the lowest so that cell-edge users cannot communicate normally. So Case Worst_UE is still not an appropriate case to further optimize the performance.

In Case Best_UE, comparing with Case 10_RB_Sep, the average throughput of eNB is reduced by $0.8 \%$, and the overall system throughput is reduced by $1.1 \%$. At the same time, the throughput of cell-edge users is increased by $7.1 \%$. So in this case, we give up a little throughput of eNB and overall system, for the purpose of cell-edge users' throughput improvement. Case Best_UE is an appropriate case to further optimize the performance.

Figure 8 shows the cellular UEs SINR of 4 optimized cases.

From this figure, we can obviously see that the cellular UEs of Case Worst_UE has the worst SINR. In Case Best_UE, due to choosing the highest transmission rate in current slot, the SINR is not as good as Case 10_ RB_Sep. But the improvement of cell-edge users' throughput is what we want.

\section{Conclusions}

Based on the simulation and analysis above, we can recognize that after introducing D2D communication into LTE-Advanced uplink system, the performance of overall system gains dramatically increase and it also improve the system spectrum efficiency. At the same time, when D2D communication reuses frequency resource

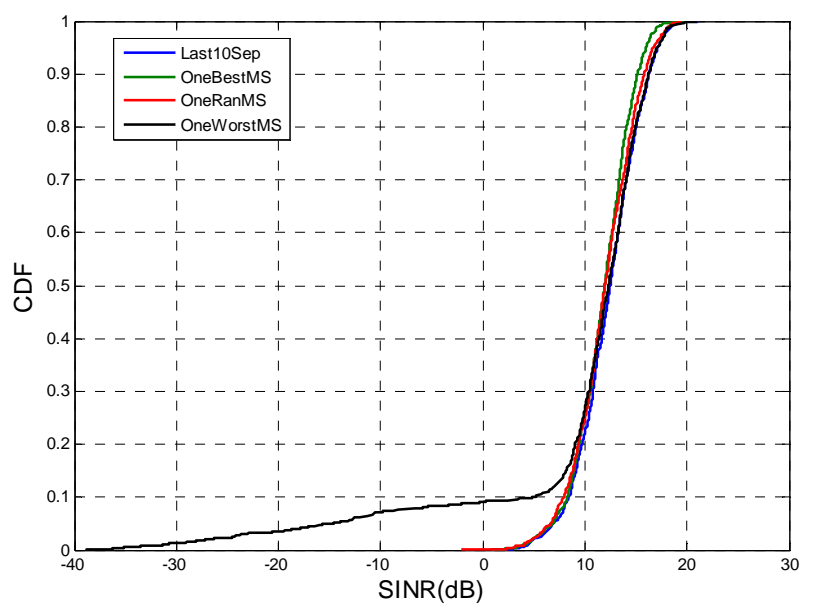

Figure 8. Cellular UEs SINR of optimized cases. with cellular users, cellular UEs will be interfered strongly. It leads to the unable transmission of cell-edge users. Therefore, proposing an appropriate resource allocation method for D2D communication is a key factor to improve the performance of the overall system. This paper firstly consider different kinds of D2D communication resource allocation methods and choose the case that D2D communication uses $10 \mathrm{RB}$ of uplink frequency resource and the cellular UEs only use another $36 \mathrm{RB}$ of uplink frequency resource, which has the best performance. On the basis of Case 10_RB_Sep, we consider choosing one cellular UE, which has the highest transmission rate in current slot, to reuse $10 \mathrm{RB}$ with $\mathrm{D} 2 \mathrm{D}$ UEs. This optimized simulation case sacrifice a little throughput of overall system in order to improve the performance of cell-edge users. After verification and analysis, Case Best_UE has the ability to improve overall system performance and limit inference for cellular communication, especially for cell-edge cellular users.

\section{Acknowledgements}

This paper is supported by National Key Technology R\&D Program of China under grant No. $2012 Z X 03003011$.

\section{REFERENCES}

[1] H. Ekstrom, A. Furusk"ar, J. Karlsson, M. Meyer, S. Parkvall, J. Torsner and M. Wahlqvist, "Solutions for the 3G Long-term Evolution,” IEEE Communications Magazine, Vol. 44, No. 3, 2006, pp. 2432-2455. doi:10.1109/MCOM.2006.1607864

[2] ITU, "ITU-R; Recommendation M. 1645 Framework and Overall Objectives of the Future Development of IMT-2000 and Systems Beyond IMT-2000,” 2003.

[3] "Estimated Spectrum Bandwidth Requirements for the Future Development of IMT-2000 and IMT-Advanced," ITU.

[4] D. C. Lee and Y. H. Kwon, "Performance Benefits of Uplink Packet Relay Protocols for Cellular-Like Systems: Quantitative Analysis," IEEE Transactions Wireless Communications, Vol. 5, No. 7, 2006, pp. 1569-1574. doi:10.1109/TWC.2006.1673062

[5] S. Haykin, "Cognitive Radio: Brain-empowered Wireless Communications," IEEE Journal on Selected Areas in Communications, Vol. 23, No. 2, 2005, pp. 201-220. doi:10.1109/JSAC.2004.839380

[6] H.-Y. Hsieh and R. Sivakumar, "On Using Peer-to-peer Communication in Cellular Wireless Data Networks," IEEE Transactions on Mobile Computing, Vol. 3, No. 1, 2004, pp. 57-72. doi:10.1109/TMC.2004.1261817

[7] P. J“anis, Y. Chia-Hao, K. Doppler, C. Ribeiro, C. Wijting, K. Hugl, O. Tirkkonen and V. Koivunen, "Device-to-device Communication Underlaying Cellular Communications Systems," Submitted to: International 
Journal of Communications, Network and System Sciences (IJCNS).

[8] V. Chandrasekhar and Z. Shen, “Optimal Uplink Power Control in Twocell Systems with Rise-over-thermal Constraints," IEEE communications letters, Vol. 12, No. 3, 2008, pp. 173-175. doi:10.1109/LCOMM.2008.071697

[9] C.-H. Yu, K. Doppler, C. Ribeiro and O. Tirkkonen, "Power Optimization of Device-to-device Communication Underlaying Cellular Communication Systems," in Proc. of IEEE International Conference on Communications, June 2009.

[10] Rep. ITU-R M.2135, “Guidelines for Evaluation of Radio Interface Technologies for IMT-Advanced,” Revision 1 to Document 5D/TEMP/90-E, 1st July 2008.

[11] C.-H. Yu, K. Doppler, C. B. Ribeiro and O. Tirkkonen, "Resource Sharing Optimization for Device-to-Device Communication Underlaying Cellular Networks. Wireless Communications," IEEE Transactions on. 8. 2011, pp. 2752-2763.

[12] M. Zulhasnine, C. C. Huang and A. Srinivasan, "Efficient Resource Allocation for Device-to-Device Communication Underlaying Lte Network," in Wireless and Mobile Computing, Networking and Communications (WiMob), 2010 IEEE 6th International Conference on, 2010), pp. 368-75. 